\title{
Impaired Memory in OT-II Transgenic Mice Is Associated with Decreased Adult Hippocampal Neurogenesis Possibly Induced by Alteration in Th2 Cytokine Levels
}

\author{
Seong Gak Jeon ${ }^{1,8}$, Kyoung Ah Kim ${ }^{1,8}$, Hyunju Chung ${ }^{2,8}$, Junghyun Choi ${ }^{2}$, Eun Ji Song ${ }^{1}$, Seung-Yun Han ${ }^{3}$, \\ Myung Sook $\mathrm{Oh}^{4}$, Jong Hwan Park ${ }^{5, *}$, Jin-il Kim ${ }^{6, *}$, and Minho Moon ${ }^{1,7, *}$
}

\begin{abstract}
Recently, an increasing number of studies have focused on the effects of CD4+ T cell on cognitive function. However, the changes of Th2 cytokines in restricted CD4+ T cell receptor (TCR) repertoire model and their effects on the adult hippocampal neurogenesis and memory are not fully understood. Here, we investigated whether and how the mice with restricted CD4+ repertoire TCR exhibit learning and memory impairment by using OT-II mice. OT-II mice showed decreased adult neurogenesis in hippocampus and short- and long- term memory impairment. Moreover, Th2 cytokines in OT-II mice are significantly increased in peripheral organs and IL-4 is significantly increased in brain. Finally, IL-4 treatment significantly inhibited the proliferation of cultured adult rat hippocampal neural stem cells. Taken together, abnormal level of Th2 cytokines can lead memory dysfunction via impaired adult neurogenesis in OT-II transgenic.
\end{abstract}

\section{INTRODUCTION}

It has traditionally been supposed that the peripheral immune

\footnotetext{
${ }^{1}$ Department of Biochemistry, College of Medicine, Konyang University, Dajeon 35365, Korea, ${ }^{2}$ Department of Core Research Laboratory, Clinical Research Institute, Kyung Hee University Hospital at Gangdong, Seoul 0527, Korea, ${ }^{3}$ Department of Anatomy, College of Medicine, Konyang University, Dajeon 35365, Korea, ${ }^{4}$ Department of Oriental Pharmaceutical Science, College of Pharmacy, Kyung Hee University, Seoul 02447, Korea, ${ }^{5}$ Laboratory Animal Medicine, College of Veterinary Medicine, Chonnam National University, Gwangju 61186, Korea, ${ }^{6}$ Department of Nursing, College of Nursing, Jeju National University, Jeju 63243, Korea, ${ }^{7}$ Konyang University Myunggok Medical Research Institute, Dajeon 35365 , Korea, ${ }^{8}$ These authors contributed equally to this work.

*Correspondence: jonpark@jnu.ac.kr (JHP); neoreva@hanmail.net (JIK); hominmoon@konyang.ac.kr (MM)
}

Received 28 March, 2016; revised 30 May, 2016; accepted 31 May, 2016; published online 19 July, 2016

Keywords: adult neurogenesis, CD4 T cells, cognition, OT-II transgenic mice, Th2 cytokines system, which is responsible for maintaining homeostasis by mediating host defense mechanisms, is isolated from the central nervous system (CNS) by the blood-brain barrier. However, many studies have shown that peripheral immune cells, such as naïve CD4+ T-cell, can directly infiltrate the blood-brain barrier or indirectly communicate across it by releasing cytokines (Bartholomaus et al., 2009; Radjavi et al., 2014a; Schwartz et al., 2013; Yirmiya and Goshen, 2011). Moreover, normal function of peripheral immune cells is critical for CNS function, especially in cognitive function. Many recent studies have indicated that the most important peripheral immune cells are the CD4+ T cell and Th2 cytokines released when a type-2 (Th2) CD 4+ T cell response occurs and Th2 cytokines have more precognitive properties than the Th1 cytokines produced by a type-1 (Th1) CD4+ T cell response (Gadani et al., 2012; Kipnis et al., 2012).

It has been reported that the dysfunction of CD4+ T cells results in a severe cognitive impairment in experimental animal models, especially in animals expressing a restricted TCR repertoire (Barnden et al., 1998; Kipnis et al., 2004; McGowan et al., 2011; Radjavi et al., 2014b; Rattazzi et al., 2013). In recent years there has been increasing evidence that impairment of CD4+ $T$ cell activation is followed by cognitive dysfunction due to down-regulated adult hippocampal neurogenesis (Wolf et al., 2009a; Ziv et al., 2006).

Adult hippocampal neurogenesis is known to be necessary for maintaining normal cognitive function. Since neurogenesis in the dentate gyrus (DG) of the hippocampus is important for both cognitive homeostasis and learning and memory (Deng et al., 2010), it seemed likely that impaired cognition when CD4+ $\mathrm{T}$ cell function was inadequate was due to a decline in hippocampal neurogenesis. As evidences that establish a relationship between peripheral immune cells and cognitive function have been accumulated, a number of studies have attempted to examine the effects of CD4+ T cells on cognitive function (Kipnis et al., 2012; Marin and Kipnis, 2013; Yirmiya and Goshen, 2011). Hippocampal neurogenesis is reportedly depressed in a transgenic animal model that either has a highly restricted CD4+ T cell receptor (TCR) repertoire or is completely immunodeficient (Ziv et al., 2006). In addition, it is reported that transgenic mice with restricted CD4+ TCR shows decreased adult hippocampal neurogenesis (Ziv et al., 2006). 
A

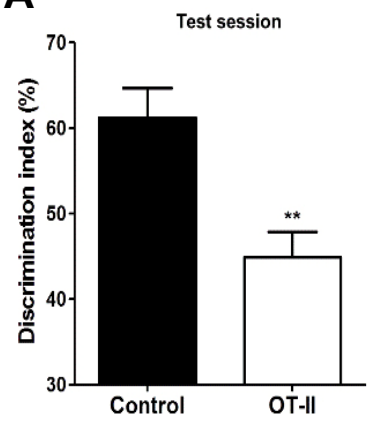

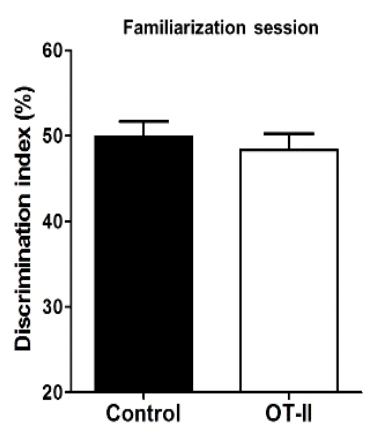

B

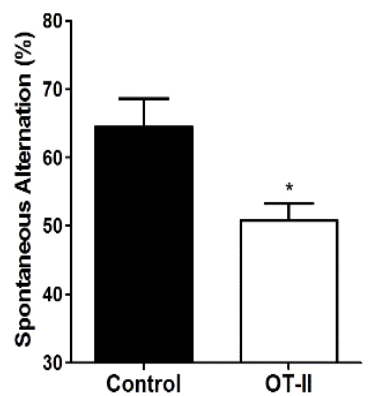

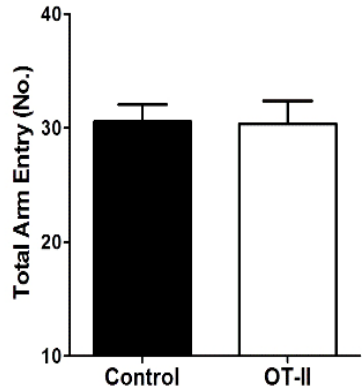

Fig. 1. Behavioral tests to determine cognitive function. (A) Novel object recognition test: Discrimination index of OT-II mice was significantly less than that for control mice in the test session, whereas discrimination indices of both groups in the familiarization ession were not different. (B) Y-maze: OT-II mice showed significantly less spontaneous alternation than control mice. Values are expressed as the mean $\pm \mathrm{SEM}{ }^{*} \mathrm{p}<0.05$, and ${ }^{* *} p<0.01$ as compared to the control mice.

However the role of $\mathrm{T}$ cell dependent cytokines in an animal model that has a restricted CD4+ TCR repertoire are not fully understood whereas the effect of changes in the level of $T$ cell dependent cytokines on hippocampal neurogenesis is well documented in the immunodeficient model.

Therefore, to expand our knowledge about the connection between the peripheral immune system and cognitive function we explored the physiological and behavioral changes that occur in CD4+ TCR restricted mice. OT-II mice express a restricted TCR repertoire whose activation depends on a non-self antigen (chicken ovalbumin) (Barnden et al., 1998). Thus, using this property of OT-II mice, we showed that abnormal Th2 cytokine levels impair the adult hippocampal neurogenesis and memory function.

\section{MATERIALS AND METHODS}

\section{Animals}

The OT-II mice have a C57BL/6 genetic background and bred in our facilities. The polymer chain reaction was used to confirm the genotypes of OT-II mice (Supplementary Fig. 1). OT-II mice overexpress TCRs consisted of $\alpha$ - and $\beta$-chain specific for chicken ovalbumin (Barnden et al., 1998; Leung et al., 2013). As a control with a normal immune system, C57BL/6 mice were purchased from Koatech (Korea) and acclimated in standard laboratory conditions for 2 weeks. All mice were male and at ages of 18 weeks, and treated in accordance with the National Institutes of Health guide for the care and use of Laboratory animals (NIH Publications No. 8023, revised 1978) and under the supervision of the Institutional Animal Care and Use Committee at Konyang University.

\section{Novel object recognition test}

The novel object recognition test (NORT) was performed in an open field box $(45 \times 45 \times 45 \mathrm{~cm})$. Prior to the test, mice were allowed a habituation period of 5 minutes in the test box without any objects for three consecutive days. After habituation, mice were placed into the test box and allowed to explore two identical objects for $3 \mathrm{~min}$. The objects used in this study were wooden blocks of the same size but of different shape (defined as a familiarization session). The $24 \mathrm{~h}$ after the familiarization session, mice were allowed to explore with one familiar object and one novel object for 3 min (defined as a test session). All sessions were recorded and analyzed using a video tracking system (EthoVision XT 10.0, Noldus Information Technology, Netherlands). The time that the mice spent exploring each of the objects, or object recognition time, was measured for each session. The object recognition time was defined as the time when mice were facing, sniffing, and biting the object or staying within $2 \mathrm{~cm}$ of it. Results were expressed as percentage of novel object recognition time [discrimination index $={ }_{t}$ novel $/$ $\left(\right.$ tnovel $\left.+{ }_{\text {tfamiliar }} \times 100\right]$.

\section{Y maze}

The Y-maze apparatus has three arms separated by $120^{\circ}$ angles (30 cm long and $8 \mathrm{~cm}$ wide with $15 \mathrm{~cm}$ high) extending from a central space $(8 \times 8 \mathrm{~cm})$. Each mouse was placed in one arm and allowed to explore freely for $5 \mathrm{~min}$ to assess their rates of spontaneous alternation. Spontaneous alternation is defined as successive entries into three different arms consecutively without repetition (i.e. ABC, BCA but not $A B A$ ). Spontaneous alternation percentage was calculated by equation [successive entries / (total arm entries - 2) $\times 100]$.

\section{Immunohistochemistry and quantification}

For immunohistochemical analysis, brain sections were rinsed briefly in phosphate buffered saline and treated with $1 \%$ hydrogen peroxide for $15 \mathrm{~min}$. The sections were incubated with Ki67 (1:2,000, abcam, England) or goat anti-doublecortin (DCX) antibody (1:1,000, Santa Cruz Biotechnology, USA) or mouse anti-synaptophysin antibody $(1: 1,000$, Sigma-Aldrich, USA) overnight at $4^{\circ} \mathrm{C}$. The sections were then incubated with biotinylated horse anti-mouse lgG or biotinylated horse anti-goat $\operatorname{lgG}$ (1:200, VECTOR, USA) and avidin-biotin-peroxidase complex solution, and then visualized with a SIGMA FAST TM $3.3^{\prime}$ Diaminobenzidine tablet (Sigma-Aldrich, USA) as a chromogen.

To quantify immunoreactivity, the images were processed and analyzed using Image-Pro Plus 6.0 program (Media Cybernetics, USA). The analysis was performed blindly in both hemispheres of four brain sections per animal. The optical densities of Ki67 and DCX in the DG and synaptophysin in the CA3-SL (cornu ammonis subfield 3 stratum lucidum) region and CA1 (cornu ammonis subfield 1) region were measured from images that were manually outlined and captured as 8-bit grayscale. 
Enzyme-linked immunosorbent assay

To quantify IL-4, IL-5, IL-6, and IL-13 levels, peripheral organs (thymus, spleen) and the brain were collected. All collected organs were homogenized and centrifuged at $4^{\circ} \mathrm{C}$ for $10 \mathrm{~min}$, respectively. The levels of Th2 cytokines in supernatants of thymus, spleen and brain were determined using commercial enzyme-linked immunosorbent assay kits (R\&D system, USA) in accordance with the manufacturer's protocol.

Adult rat hippocampal NSC cultures and treatments

Adult rat hippocampal neural stem cells (NSCs) were obtained from Chemicon (Catalog No. SCR022, USA). These cells are ready-to-use primary NSCs isolated from the hippocampus of adult Fisher 344 rats. They were grown in a NSC expansion medium containing Dulbecco's modified Eagles's medium (DMEM)/F12 medium (Gibco/Invitrogen, USA) with L-glutamine, B27 supplement, $1 \times$ solution of penicillin, streptomycin and fungizone, and basic FGF (bFGF, $20 \mathrm{ng} / \mathrm{ml}$ ). Tissue culture plastic- or glasswares that were used to culture hippocampal NSCs were coated with poly-L-ornithine $(10 \mu \mathrm{g} / \mathrm{ml})$ and laminin $(5 \mu \mathrm{g} / \mathrm{ml})$. The hippocampal NSCs were maintained at $37^{\circ} \mathrm{C}$ in a 5\% CO2 humidified incubator and passaged once every 3-4 days.

\section{Cell counting kit-8 (CCK) analysis}

For the evaluation of effect of IL-4 on cell proliferation, the proliferation index of each group was determined using the CCK-8 method (Enzo life science Inc., Switzerland) according to the manufacturer's instructions. Cells in the exponential phase of growth were seeded in 24-well plates at a density of $3 \times 10^{5}$ cells/well, and cultured in F12/DMEM (500 $\mu$ l) supplemented with $0.1 \%$ B27 (24 wells per group). In brief, $20 \mu$ l of CCK- 8 solution was added into each well (containing $200 \mu \mathrm{l}$ of medium), and further cultured for $2 \mathrm{~h}$ at $37^{\circ} \mathrm{C}$. The absorbance of each group at $450 \mathrm{~nm}$ was detected $(\mathrm{n}=3)$ using an absorbance microplate reader (Molecular devices LLC., USA) and it was directly proportional to the number of living cells. Recombinant rat interleukin (IL)-4 were obtained from PEPROTECH (USA).

\section{Western blot analysis}

To confirm the effect of IL-4 on proliferation of NSCs and to investigate its the mechanism, the expression of proliferating cell nuclear antigen (PCNA) using western blot analysis. Cells were lysed in a buffer containing $20 \mathrm{mM}$ Tris- $\mathrm{HCl}(\mathrm{pH} 7.4), 1$ $\mathrm{mM}$ EDTA, $140 \mathrm{mM} \mathrm{NaCl}, 1 \%(\mathrm{w} / \mathrm{v})$ Nonidet P-40, $1 \mathrm{mM}$ $\mathrm{Na}_{3} \mathrm{VO}_{4}, 1 \mathrm{mM}$ phenylmethylsulfonyl fluoride, $50 \mathrm{mM} \mathrm{NaF}$, and $10 \mu \mathrm{g} / \mathrm{ml}$ aprotinin. Cell lysates were separated by $12 \%$ SDSpolyacrylamide gel electrophoresis, and electrotransferred to a polyvinylidene difluoride membrane (Bio-Rad). The membranes were incubated with blocking buffer ( $1 \times$ Tris-buffered saline, $1 \%$ $\mathrm{BSA}, 1 \%$ nonfat dry milk) for $1 \mathrm{~h}$ and then incubated with the primary antibody against PCNA (Santa Cruz Biotechnology; $1: 1,000)$ for overnight at $4^{\circ} \mathrm{C}$. Primary antibodies were visualized with peroxidase-conjugated anti-rabbit IgG and a chemiluminescent detection system (Santa Cruz Biotechnology, Inc., USA). The ChemicDoc XRS system (Bio-Rad) was used for visualization and quantification using Quantity One imaging software (Bio-Rad).

\section{Statistical analysis}

All data are shown as mean \pm SEM. Statistical differences between groups were analyzed by independent T-test using SigmaStat for Windows Version 3.10 (Systat Software, Inc., USA). A $p$-value $<0.05$ was considered statistically significant.

\section{RESULTS}

Impaired memory functions in OT-II mice

A number of previous studies demonstrated that impaired or altered TCR may related to dysfunction of memory and learning especially for spatial memory (Brynskikh et al., 2008; Kipnis et al., 2004; Wolf et al., 2009a; Yirmiya and Goshen, 2011). To address whether OT-II mice maintained normal cognitive function or not, we examined cognitive function by using two separate sets of behavioral tests. First, we conducted NORT to evaluate hippocampal-related long-term recognition memory (Antunes and Biala, 2012; Bevins and Besheer, 2006; Broadbent et al., 2009; Choi et al., 2011; Taglialatela et al., 2009). There was no difference in exploration time between OT-II mice and control mice during the familiarization session. In the test session, control mice spent more time with the novel object than did the OT-II mice, indicating that OT-II mice had less curiosity (Fig. 1A). Secondly, we performed the Y-maze test to assess hippocampal-related short-term and spatial memory by evaluating spontaneous alternations (Bannerman et al., 2014; Moon et al., 2013). OT-II mice had a lower percentage of spontaneous alternations than control mice while the number of total arm entries was similar, suggesting no differences in motor function (Fig. 1B). These findings demonstrate that OT-II mice showed an obvious impairment of shortand long-term memory as well as spatial memory.

\section{Down-regulated adult hippocampal neurogenesis and} impairment of synaptic integration in OT-II mice

There had been strong evidence that the peripheral immune system including CD4+ T cells is highly associated with adult hippocampal neurogenesis (Kipnis et al., 2004; Wolf et al., 2009a; 2009b). To determine whether the limited TCR diversity of OT-II mice inhibits adult hippocampal neurogenesis, we conducted Ki67 and DCX staining and counted Ki67-, and DCXpositive cells in the DG. Ki67 is expressed during mitosis and is a marker of cell proliferation. In addition, DCX is expressed in immature neuroblast and used as a marker for adult neurogenesis (Lee et al., 2015; Ouchi et al., 2013). Compared to control mice, the number of Ki67-positive cells in the DG of the OT-II mice is significantly diminished (Fig. $2 \mathrm{~A}$ ), as is the number of DCX-positive immature neurons (Fig. 2B). Following neurogenesis, new dentate granule cells extend their axons and form synapses on CA3-SL pyramidal neurons (Goshen et al., 2008). As a marker of presynaptic marker, we used synaptophysin staining to assess synaptic density in the CA3-SL region of the hippocampus. OT-II mice showed a significant reduction of synaptic density in the CA3-SL region than did control mice (Fig 2C). We further performed additional synaptophysin staining of CA1 region to identify whether behavioral changes are mediated by changes of synaptic formation in other region of hippocampus since behavioral tests such as Y-maze and NORT that indicate spatial memory and recognition memory are related to CA1 region as well (Ainge et al., 2007; Bannerman et al., 2014; Rampon et al., 2000; Tsien et al., 1996). There was no difference of synaptic density in the CA1 region between OT-II mice and control mice (Fig. 2D). These findings strongly indicate that OT-II transgenic mice have a down-regulation of adult hippocampal neurogenesis and impairment in synaptic integration.

Increased level of peripheral and central Th2 T cell releasing cytokines in OT-II TCR mice

Many recent studies have suggested that down-regulated adult neurogenesis and neural integration in immune-compromised 
A
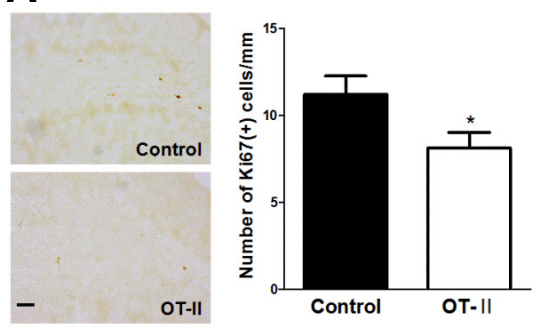

C
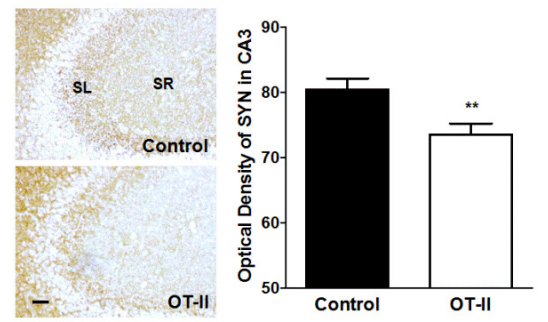

B

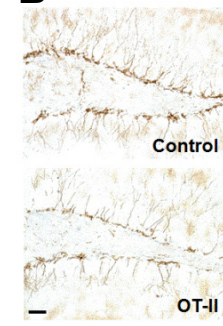

D
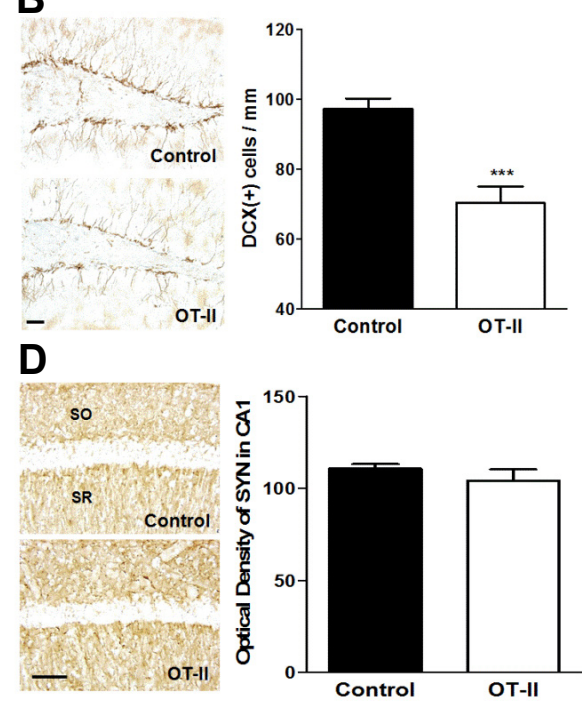

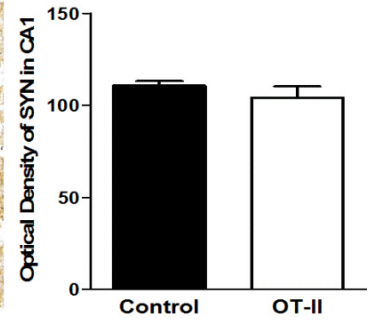

Fig. 2. OT-II mice showed decreased neurogenesis and synaptic density in hippocampus. (A) Representative images of Ki67 staining of the DG. (B) DCX-positive cells were significantly less abundant in OT-II mice than in control mice. (C) Immunoreactivity of synaptophysin in the CA3 subfields (SL: stratum lucidum; SR: stratum radiatum) of the hippocampus was markedly less abundant in OT-II mice than in the control mice. (D) Immunoreactivity of synaptophysin in the CA1 subfields (SR: stratum radiatum; SO: stratum oriens) of the hippocampus. There was no difference between OT-II mice and control mice. Values are expressed as the mean \pm SEM ${ }^{*} p<0.05,{ }^{* *} p<0.01$, and ${ }^{* * *} p<0.001$ as compared to the control mice. Scale bar $=50 \mu \mathrm{m}$.
A

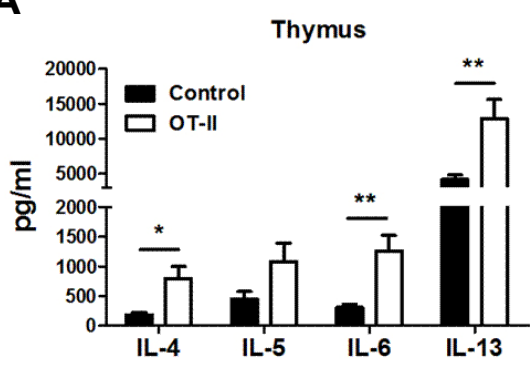

B

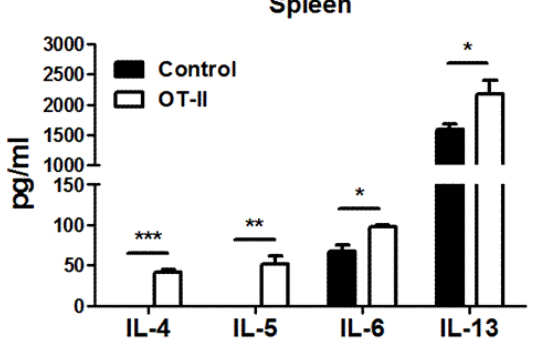

C

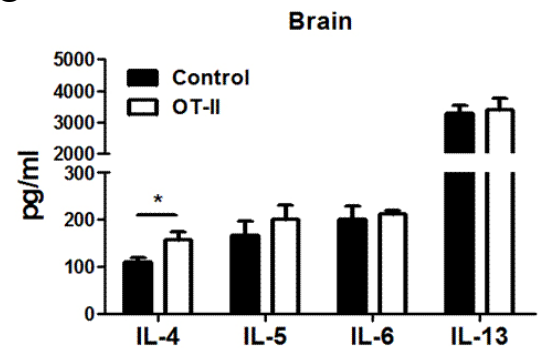

Fig. 3. Altered levels of Th2 cytokines in OT-II mice. (A, B) Levels of peripheral Th2 cytokines released by naïve ovalbumin-specific T cells were significantly higher in OT-II mice than for control mice. (C) OT-II mice expressed significantly higher level of IL-4 in the brain than control mice. Values are expressed as the mean \pm SEM ${ }^{*} p<0.05,{ }^{* *} p<0.01$, and ${ }^{* * *} p<0.001$ as compared to the control mice.

status is highly associated with the release of cytokines by Th2 T cells (Derecki et al., 2010; Kipnis et al., 2004; 2012; McGowan et al., 2011). Therefore, it is presumed that impaired cognitive function in OT-II mice is mediated by an altered level of Th2 cytokines, because of its limited TCR repertoire. To clarify the factors meditating reduced adult hippocampal neurogenesis in animals with a limited TCR, we examined the level of Th2 cytokines in the spleen, thymus, and brain. OT-II mice expressed higher levels of all Th2 cytokines in the spleen and of IL-4 in the brain than control mice (Fig. 3). Thus, these findings demonstrate a detrimental mediating effect of elevated levels of Th2 cytokines in the periphery and in the CNS on neurogenesis and synaptic integration.

\section{IL-4-induced inhibition of proliferation of adult rat hippocampal NSCs}

To investigate the direct effect of IL-4 on adult hippocampal neurogenesis, we next determine whether IL-4 can inhibit the proliferation of adult hippocampal NSCs in vitro using CCK-8. The addition of IL-4 $(5,10,20$, and $50 \mathrm{ng} / \mathrm{ml})$ to NSCs for 6,24 , 48 , and $72 \mathrm{~h}$ inhibited the proliferation in a time-dependent manner (Fig. 4A). As shown in Fig. 4B, incubation of cells with 10 $\mathrm{ng} / \mathrm{mg} \mathrm{IL-4}$ resulted in a time-dependent reduction of cell proliferation of adult hippocampal NSCs. This data suggests that IL-4 directly reduces the generation of newborn neurons from NSCs.

\section{Inhibitory effects of IL-4 on proliferation of NSCs via reducing the expression of PCNA}

To confirm the effect of IL-4 on proliferation of NSCs and to investigate its mechanism, we performed immunoblotting analysis with proliferating cell nuclear antigen (PCNA) which is a marker of NSC-specific cell proliferation during S-phase (Arai et al., 2011). Adult hippocampal NSCs were treated with $10 \mathrm{ng} / \mathrm{ml}$ of IL-4 and the expression of PCNA was examined using western blot analysis. Adult hippocampal NSCs with 24 hours treatment of IL-4 showed significantly decreased PCNA levels compared to the control (Fig. 5). This result shows that IL-4-induced inhibition of proliferation of adult rat hippocampal NSCs may be mediated by down-regulation of PCNA.

\section{DISCUSSION}

In the present study, we performed behavioral tests to examine the difference in memory function between OT-II mice and 

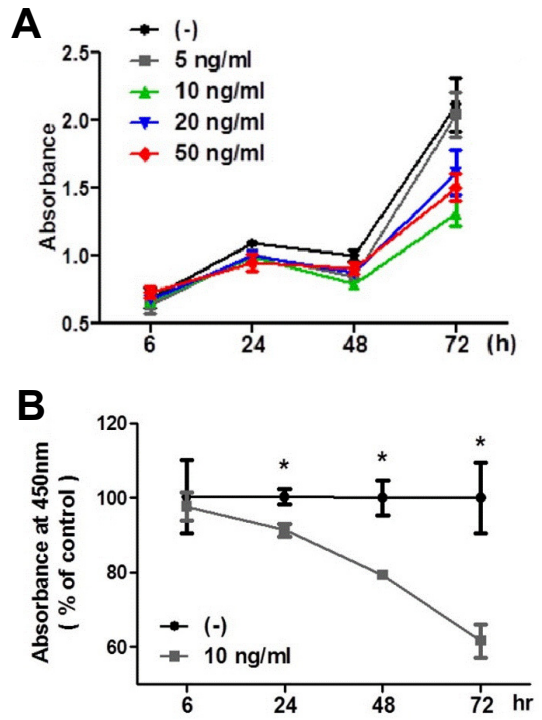

Fig. 4. Effects of IL-4 on the proliferation of cultured adult rat hippocampal NSCs. (A) Dose-dependency and time-course of IL-4induced inhibition of cell proliferation. (B) NSCs were treated with $10 \mathrm{ng} / \mathrm{ml} \mathrm{IL}-4$ for $6,24,48$, and $74 \mathrm{~h}$. Data are mean \pm SEM. ${ }^{*} p<$ 0.05 versus vehicle-treated cells.

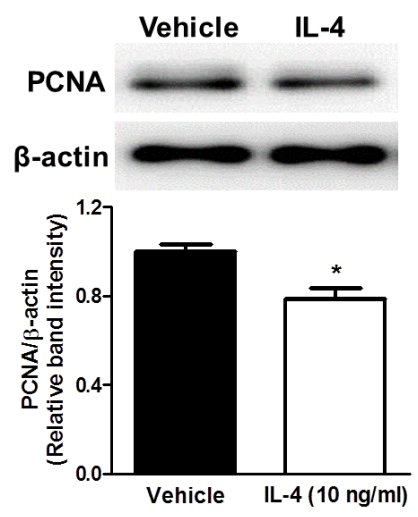

Fig. 5. Confirmation of inhibitory effect of IL-4 on the proliferation of cultured adult rat hippocampal NSCs. Band intensity of western blot indicated that the expression of PCNA in adult rat hippocampal NPCs was reduced after treatment of $10 \mathrm{ng} / \mathrm{ml}$ of IL-4 for $24 \mathrm{~h}$. Data are mean \pm SEM. ${ }^{*} p<0.05$ versus vehicle-treated cells.

normal control mice. As expected, OT-II mice showed significant learning and memory impairment. These data indicating memory impairments in OT-II mice are consistent with previous studies in which mice with inhibited or transformed $\mathrm{T}$ cells showed significantly impairments of learning and memory (Cushman et al., 2003; Brynskikh et al., 2008; Kipnis et al., 2004). Since changes in behavior, such as decreased spontaneous alternation and novel object recognition time indicate short-, long-term memory and spatial memory impairment (Bevins and Besheer, 2006; Choi et al., 2011; Moon et al., 2013). It can be concluded that intact adaptive peripheral immunity is critical for learning and memory function.

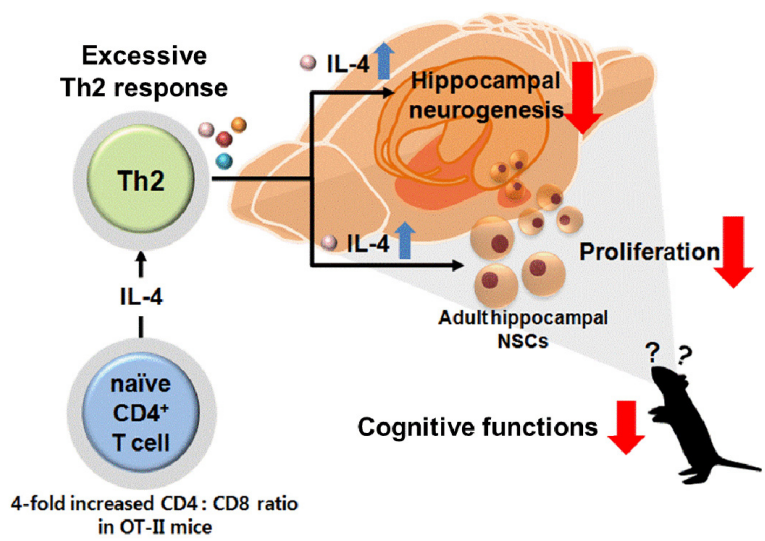

Fig. 6. The proposed mechanisms for how excessive Th2 cytokines can impair the cognitive function in OT-II mice.

Despite the growing evidences of the roles of CD4+ T cells on cognitive function, one of the main remaining questions regarding the declined cognitive function in restricted CD4+ TCR diversity is whether the Th2 cytokines released by CD4+ T cells are involved in the impairment of cognitive function. Using OT-II mice, we confirmed that a significant impairment of learning and memory function occurs in mice with a restricted CD4+ TCR repertoire with changed levels of Th2 cytokine.

Many previous studies identified adult neurogenesis as critical for altered cognitive behaviors in adaptive immune cell disorders (Goshen et al., 2008; McGowan et al., 2011; Wolf et al., 2009a; Ziv et al., 2006). To examine the neurostructural mechanisms of impaired cognitive function in OT-II mice, we investigated changes in the DG where adult neurogenesis takes place and $\mathrm{CA} 3$ regions where synaptic integration of newly generated neurons occurs. We observed significant declines in new born cells stained for Ki67 and DCX in the DG. The expression of synaptophysin also was reduced in the CA3 subfields but not in other region such as CA1 region. This implied that impaired learning and memory function in OT-II mice was a consequence of decreased adult hippocampal neurogenesis in the DG and reduced its synaptic integration in the CA3 regions.

These data can be explained by an emerging conceptual model called a computational model of neurogenesis. In the point of computation model of neurogenesis, the long-term retention of memory requires the addition of new neurons and the synaptic integration of new neurons with existing neurons (Becker et al., 2009; Deng et al., 2010; Ming and Song, 2005). However, there have been controversial reports that decreased hippocampal neurogenesis also results in impairment of shortterm memory. There are also previous reports in which decreased hippocampal neurogenesis does not result in shortterm memory impairment. The findings of the present study implying impaired short-term memory are consistent with theformer set of results and with a previous study in which p50 knockout mice showed both significantly impaired short-term memory and decreased hippocampal neurogenesis (Goshen et al., 2008). Moreover, the Hebbian theory supports the expectation that impaired hippocampal neurogenesis could be expected to produce short-term memory impairment. Thus, it is reasonable that reduced short- and long-term memory in OT-II mice is due to decreased proliferation, differentiation of new neurons and synaptic integration. 
Next, we measured Th2 cytokines; IL-4, IL-5, IL-6, and IL-13 to verify whether $\mathrm{T}$ cell dependent cytokines participate in adult neurogenesis in a restricted CD4+ TCR repertoire model. The activation of $T$ cells requires the recognition of an antigen as presented by major histocompatibility complex II molecules on the surfaces of the antigen presenting cells that trigger the immune system into action (Banchereau and Steinman, 1998; Furuta et al., 2012). OT-II mice that mainly bear non-self antigen-specific CD4+ TCRs which need ovalbumin to become activated (Barnden et al., 1998; Kipnis et al., 2012; Ziv et al., 2006). The CD4+ T cells of OT-II mice cannot recognize antigens other than ovalbumin, and therefore should not have been activated in animals lacking prior exposure to this substance. Surprisingly, the OT-II mice used in the present study showed a significantly increased level of all Th2 cytokines in the periphery and of interleukin-4 (IL-4) in the CNS. Since these OT-II mice had not had prior contact with ovalbumin, they should have had naïve CD4+ T cells.

The increased levels of Th2 cytokines that OT-II mice showed in both the periphery and the CNS could not have been induced by activation of the CD4+ T cells by exposure to ovalbumin. No previous study has examined the level of cytokines released by naïve CD4+ T cells in OT-II mice. However, the observed increase in the level of Th2 cytokines may be explainable due to the fact that OT-II mice exhibit a four-fold increase in the ratio of CD4 to CD8 over control mice (Leung et al., 2013; Palumbo et al., 2010). There are studies suggesting that Th2 cytokine response can be initiated by endogenous IL-4 released from naïve CD4+ T cells (Halim et al., 2014; NobenTrauth et al., 2000; 2002) as well as IL-4 can be released from naïve CD4+ T cells (Bullens et al., 1999). Moreover, our data are consistent with a previous study showing that patients with higher CD4/CD8 ratio showed increased IL-4 levels in peripheral blood and CD4/CD8 ratio is more important than the number of CD4+ T cells in the production of IL-4 (Lee et al., 2001). In this regard, neuronal cells have IL-4 receptors (IL-4R $\alpha$ ) (Gadani et al., 2012) and IL-4 in OT-II mice is constitutive likewise IL-4 transgenic mice, it can be explained that increased IL4 may affected down-regulated proliferation of progenitor cells in hippocampus and impaired cognitive functions. Based on these previous studies, our results showed increased level of IL-4 in both periphery and brain may due to the release of Th2 cytokines by naïve CD4+ T cells which are four-fold increased ratio in OT-II mice.

We further investigate the direct effect of IL- 4 on proliferation of NSCs using CCK-8 analysis. In in vitro CCK-8 analysis, it is shown that IL-4 directly inhibits the proliferation of NSCs. To address the underlying mechanism of IL-4-induced inhibition of proliferation of adult rat hippocampal NSCs, we performed the immunoblotting analysis of PCNA expression. NSCs with IL-4 showed significant decreased expression of PCNA than vehicle treated NSCs. The PCNA expression analysis data are in part consistent with a report that the level of IL-6, one of the Th2 cytokines, is correlated with PCNA levels in advancing disease stage (Tsirakis et al., 2011). PCNA is a biomarker of NSCspecific cell proliferation as well as is needed to DNA replication in S-phase of adult hippocampal NSCs (Arai et al., 2011; Mandyam et al., 2007; Paunesku et al., 2001). It is well known that PCNA is not found in normal neurons but is found in NSCs (Hoglinger et al., 2007; Sierra et al., 2011). PCNA has various functions in the cell cycle. It acts as a factor for DNA polymerase during DNA replication. It also controls the cell cycle and repair of DNA damage (Paunesku et al., 2001; Zhang et al., 2014). Thus, decreased expression of PCNA may, at least, be associated with IL-4-induced inhibition of proliferation of adult rat hippocampal NSCs by alteration of DNA replication during S-phase. Further in vivo studies are needed to elucidate the effects of IL-4 on DG neurogenesis and their specific mechanisms.

IL-4, a Th2 cytokine secreted by CD4 T cell engages various biological roles including adaptive immune response and even cognition function. Many studies have described beneficial effects of IL-4 on cognitive functions (Derecki et al., 2010; Gadani et al., 2012; Kipnis et al., 2012). However, harmful effects of IL-4 also have reported. It has been shown that upregulation of IL-4 induces reactive oxidative stress (ROS) production by activating microglial NADPH oxidase in cells from various systems (Harper et al., 2005; Park et al., 2008; Walch et al., 2006). Moreover, increased IL-4 level in hippocampus induced by thrombin administration leads to degeneration of CA1 region in hippocampus (Park et al., 2008). With respect to studies on the effects of IL-4 on CNS, constitutive IL-4 inhibits the proliferation of retinal progenitor cell which is a part of the CNS (Serre et al., 2010). Another studies have reported that there was overexpressed IL-4 in the experimental autoimmune encephalomyelitis model (Khoury et al., 1992; Lafaille et al., 1997). Interestingly, experimental autoimmune encephalomyelitis did not appear in IL-4 knockout animal (Lafaille et al., 1997). These indicate that normal expression of IL-4 is important to regulate adaptive immune functions including maintaining a CNS homeostasis. Moreover, transgenic mice that overexpressing IL-4 showed autoimmune-like disorders; anemia, glomerulonephritis and arthritis (Erb et al., 1997; Rivas et al., 1995). Another study has revealed that the relationship between asthma and cognition. Asthma is the risk factor of cognitive impairment in older people (Caldera-Alvarado et al., 2013). Although, in that study the level of IL-4 was not measured, the IL-4 is highly associated with asthma. Indeed, up-regulated Th2 cytokines have been observed in allergic immune response like asthma. IL-4 and IL-13 have a critical role in asthma which is chronic allergic inflammatory disease (Brusselle et al., 1994; Chatila, 2004; Tavakkol Afshari et al., 2007). Taken together, our data and previous reports suggest that overexpression of IL-4 reduces neurogenesis and synaptic formation in hippocampus.

Additional possibility can be raised by previous studies reporting down-regulated neurogenesis can affected by elevated levels of other Th2 cytokines. Many studies demonstrated that Th2 cytokines can suppress the hippocampal neurogenesis by regulating hypothalamic-pituitary-adrenal (HPA) axis (Schloesser et al., 2009; Snyder et al., 2011). Thus, the down-regulated adult hippocampal neurogenesis and abnormal cognitive behaviors may due to an activation of HPA axis induced by elevated Th2 cytokine levels in peripheral organs.

Using mice with a restricted CD4+ TCR repertoire, we have examined the role of Th2 cytokines on adult hippocampal neurogenesis and memory function. Overexpression of Th2 cytokines may lead to an alteration of neurogenesis and cognitive behavior by increased IL-4 level in the brain and other Th2 cytokines in periphery (Fig. 6). Taken together, it appears that maintaining a normal level of peripheral Th2 cytokines may be a possible strategy for maintaining normal memory function.

Note: Supplementary information is available on the Molecules and Cells website (www.molcells.org).

\section{ACKNOWLEDGMENTS}

This work was supported by Konyang University Myunggok Research Fund of 2015. 


\section{REFERENCES}

Ainge, J.A., Tamosiunaite, M., Woergoetter, F., and Dudchenko, P.A. (2007). Hippocampal CA1 place cells encode intended destination on a maze with multiple choice points. J. Neurosci. 27, 9769-9779.

Antunes, M., and Biala, G. (2012). The novel object recognition memory: neurobiology, test procedure, and its modifications. Cogn. Process 13, 93-110.

Arai, Y., Pulvers, J.N., Haffner, C., Schilling, B., Nusslein, I., Calegari, F., and Huttner, W.B. (2011). Neural stem and progenitor cells shorten S-phase on commitment to neuron production. Nat. Commun. 2, 154.

Banchereau, J., and Steinman, R.M. (1998). Dendritic cells and the control of immunity. Nature 392, 245-252.

Bannerman, D.M., Sprengel, R., Sanderson, D.J., McHugh, S.B., Rawlins, J.N., Monyer, H., and Seeburg, P.H. (2014). Hippocampal synaptic plasticity, spatial memory and anxiety. Nat. Rev. Neurosci. 15, 181-192.

Barnden, M.J., Allison, J., Heath, W.R., and Carbone, F.R. (1998). Defective TCR expression in transgenic mice constructed using cDNA-based alpha- and beta-chain genes under the control of heterologous regulatory elements. Immunol. Cell Biol. 76, 34-40.

Bartholomaus, I., Kawakami, N., Odoardi, F. Schlager, C. Milikovic, D., Ellwart, J.W., Klinkert, W.E., Flugel-Koch, C., Issekutz, T.B., Wekerle, H., et al. (2009). Effector T cell interactions with meningeal vascular structures in nascent autoimmune CNS lesions. Nature 462, 94-98.

Becker, S., Macqueen, G., and Wojtowicz, J.M. (2009). Computational modeling and empirical studies of hippocampal neurogenesis-dependent memory: Effects of interference, stress and depression. Brain Res. 24, 45-54.

Bevins, R.A., and Besheer, J. (2006). Object recognition in rats and mice: a one-trial non-matching-to-sample learning task to study 'recognition memory'. Nat. Protoc. 1, 1306-1311.

Broadbent, N.J., Gaskin, S., Squire, L.R., and Clark, R.E. (2009). Object recognition memory and the rodent hippocampus. Learn. Mem. 17, 5-11.

Brusselle, G.G., Kips, J.C., Tavernier, J.H., van der Heyden, J.G., Cuvelier, C.A., Pauwels, R.A., and Bluethmann, H. (1994). Attenuation of allergic airway inflammation in IL-4 deficient mice. Clin. Exp. Allergy 24, 73-80.

Brynskikh, A., Warren, T., Zhu, J., and Kipnis, J. (2008). Adaptive immunity affects learning behavior in mice. Brain Behav. Immun. 22, 861-869.

Bullens, D.M., Rafiq, K., Kasran, A., Van Gool, S.W., and Ceuppens, J.L. (1999). Naive human T cells can be a source of IL-4 during primary immune responses. Clin. Exp. Immunol. 118, 384-391.

Caldera-Alvarado, G., Khan, D.A., Defina, L.F., Pieper, A., and Brown, E.S. (2013). Relationship between asthma and cognition: the Cooper Center Longitudinal Study. Allergy 68, 545-548.

Chatila, T.A. (2004). Interleukin-4 receptor signaling pathways in asthma pathogenesis. Trends Mol. Med. 10, 493-499.

Choi, J.G., Moon, M., Jeong, H.U., Kim, M.C., Kim, S.Y., and Oh, M.S. (2011). Cistanches Herba enhances learning and memory by inducing nerve growth factor. Behav. Brain Res. 216, 652-658.

Cushman, J., Lo, J., Huang, Z., Wasserfall, C., and Petitto, J.M. (2003). Neurobehavioral changes resulting from recombinase activation gene 1 deletion. Clin. Diagn. Lab. Immunol. 10, 13-18.

Deng, W., Aimone, J.B., and Gage, F.H. (2010). New neurons and new memories: how does adult hippocampal neurogenesis affect learning and memory? Nat. Rev. Neurosci.11, 339-350.

Derecki, N.C., Cardani, A.N., Yang, C.H., Quinnies, K.M., Crihfield, A., Lynch, K.R., and Kipnis, J. (2010). Regulation of learning and memory by meningeal immunity: a key role for IL-4. J. Exp. Med. 207, 1067-1080.

Erb, K.J., Ruger, B., von Brevern, M., Ryffel, B., Schimpl, A., and Rivett, K. (1997). Constitutive expression of interleukin (IL).-4 in vivo causes autoimmune-type disorders in mice. J. Exp. Med. 185, 329-339.

Furuta, K., Ishido, S., and Roche, P.A. (2012). Encounter with antigen-specific primed CD4 T cells promotes MHC class II degradation in dendritic cells. Proc. Natl. Acad. Sci. USA 109, 19380-19385.

Gadani, S.P., Cronk, J.C., Norris, G.T., and Kipnis, J. (2012). IL-4 in the brain: a cytokine to remember. J. Immunol.189, 4213-4219.
Goshen, I., Kreisel, T., Ben-Menachem-Zidon, O., Licht, T., Weidenfeld, J., Ben-Hur, T., and Yirmiya, R. (2008). Brain interleukin-1 mediates chronic stress-induced depression in mice via adrenocortical activation and hippocampal neurogenesis suppression. Mol. Psychiatry 13, 717-728.

Halim, T.Y., Steer, C.A., Matha, L., Gold, M.J., Martinez-Gonzalez, I., McNagny, K.M., McKenzie, A.N., and Takei, F. (2014). Group 2 innate lymphoid cells are critical for the initiation of adaptive $T$ helper 2 cell-mediated allergic lung inflammation. Immunity 40, 425-435.

Harper, R.W., Xu, C., Eiserich, J.P., Chen, Y., Kao, C.Y., Thai, P., Setiadi, H., and Wu, R. (2005). Differential regulation of dual NADPH oxidases/peroxidases, Duox1 and Duox2, by Th1 and Th2 cytokines in respiratory tract epithelium. FEBS Lett. 579, 4911-4917.

Hoglinger, G.U., Breunig, J.J., Depboylu, C., Rouaux, C., Michel, P.P., Alvarez-Fischer, D., Boutillier, A.L., Degregori, J., Oertel, W.H., Rakic, P., et al. (2007). The pRb/E2F cell-cycle pathway mediates cell death in Parkinson's disease. Proc. Natl. Acad. Sci. USA 104, 3585-3590.

Khoury, S.J., Hancock, W.W., and Weiner, H.L. (1992). Oral tolerance to myelin basic protein and natural recovery from experimental autoimmune encephalomyelitis are associated with downregulation of inflammatory cytokines and differential upregulation of transforming growth factor beta, interleukin 4, and prostaglandin E expression in the brain. J. Exp. Med. 176, 13551364.

Kipnis, J., Cohen, H., Cardon, M., Ziv, Y., and Schwartz, M. (2004). $T$ cell deficiency leads to cognitive dysfunction: implications for therapeutic vaccination for schizophrenia and other psychiatric conditions. Proc. Natl. Acad. Sci. USA 101, 8180-8185.

Kipnis, J., Gadani, S., and Derecki, N.C. (2012). Pro-cognitive properties of T cells. Nature reviews. Immunology 12, 663-669.

Lafaille, J.J., Keere, F.V., Hsu, A.L., Baron, J.L., Haas, W., Raine, C.S., and Tonegawa, S. (1997). Myelin basic protein-specific T helper 2 (Th2). cells cause experimental autoimmune encephalomyelitis in immunodeficient hosts rather than protect them from the disease. J. Exp. Med. 186, 307-312.

Lee, S.Y., Kim, S.J., Kwon, S.S., Kim, Y.K., Kim, K.H., Moon, H.S., Song, J.S., and Park, S.H. (2001). Distribution and cytokine production of CD4 and CD8 T-lymphocyte subsets in patients with acute asthma attacks. Ann. Allergy Asthma Immunol. 86, 659-664.

Lee, W., Moon, M., Kim, H.G., Lee, T.H., and Oh, M.S. (2015). Heat stress-induced memory impairment is associated with neuroinflammation in mice. J. Neuroinflammation 12, 015-0324.

Leung, S., Smith, D., Myc, A., Morry, J., and Baker, J.R., Jr. (2013). OT-II TCR transgenic mice fail to produce anti-ovalbumin antibodies upon vaccination. Cell Immunol. 282, 79-84.

Mandyam, C.D., Harburg, G.C., and Eisch, A.J. (2007). Determination of key aspects of precursor cell proliferation, cell cycle length and kinetics in the adult mouse subgranular zone. Neuroscience 146, 108-122.

Marin, I., and Kipnis, J. (2013). Learning and memory ... and the immune system. Learn. Mem. 20, 601-606.

McGowan, P.O., Hope, T.A., Meck, W.H., Kelsoe, G., and Williams, C.L. (2011). Impaired social recognition memory in recombination activating gene 1-deficient mice. Brain Res. 1383, 187-195.

Ming, G.L., and Song, H. (2005). Adult neurogenesis in the mammalian central nervous system. Annu. Rev. Neurosci. 28, 223-250.

Moon, M., Song, H., Hong, H.J., Nam, D.W., Cha, M.Y., Oh, M.S., Yu, J., Ryu, H., and Mook-Jung, I. (2013). Vitamin D-binding protein interacts with Abeta and suppresses Abeta-mediated pathology. Cell. Death Differ. 20, 630-638.

Noben-Trauth, N., Hu-Li, J., and Paul, W.E. (2000). Conventional, naive CD4+ T cells provide an initial source of IL-4 during Th2 differentiation. J. Immunol.165, 3620-3625.

Noben-Trauth, N., Hu-Li, J., and Paul, W.E. (2002). IL-4 secreted from individual naive CD4+ T cells acts in an autocrine manner to induce Th2 differentiation. Eur. J. Immunol. 32, 1428-1433.

Ouchi, Y., Banno, Y., Shimizu, Y., Ando, S., Hasegawa, H., Adachi, K., and Iwamoto, T. (2013). Reduced adult hippocampal neurogenesis and working memory deficits in the Dgcr8-deficient mouse model of 22q11.2 deletion-associated schizophrenia can be rescued by IGF2. J. Neurosci. 33, 9408-9419. 
Palumbo, M.L., Canzobre, M.C., Pascuan, C.G., Rios, H., Wald, M., and Genaro, A.M. (2010). Stress induced cognitive deficit is differentially modulated in BALB/c and C57BI/6 mice: correlation with Th1/Th2 balance after stress exposure. J. Neuroimmunol. 218, 12-20.

Park, K.W., Baik, H.H., and Jin, B.K. (2008). Interleukin-4-induced oxidative stress via microglial NADPH oxidase contributes to the death of hippocampal neurons in vivo. Curr. Aging Sci. 1, 192201.

Paunesku, T., Mittal, S., Protic, M., Oryhon, J., Korolev, S.V. Joachimiak, A., and Woloschak, G.E. (2001). Proliferating cell nuclear antigen (PCNA).: ringmaster of the genome. Int J. Radiat. Biol. 77, 1007-1021.

Radjavi, A., Smirnov, I., Derecki, N., and Kipnis, J. (2014a). Dynamics of the meningeal CD4(+). T-cell repertoire are defined by the cervical lymph nodes and facilitate cognitive task performance in mice. Mol. Psychiatry 19, 531-533.

Radjavi, A., Smirnov, I. and Kipnis, J. (2014b). Brain antigenreactive CD4+ T cells are sufficient to support learning behavior in mice with limited T cell repertoire. Brain Behav. Immun. 35, 5863

Rampon, C., Tang, Y.P., Goodhouse, J., Shimizu, E., Kyin, M., and Tsien, J.Z. (2000). Enrichment induces structural changes and recovery from nonspatial memory deficits in CA1 NMDAR1knockout mice. Nat. Neurosci. 3, 238-244.

Rattazzi, L., Piras, G., Ono, M., Deacon, R., Pariante, C.M., and D'Acquisto, F. (2013). CD4(+). but not CD8(+). T cells revert the impaired emotional behavior of immunocompromised RAG-1deficient mice. Transl. Psychiatry 3, e280.

Rivas, D., Mozo, L., Zamorano, J., Gayo, A., Torre-Alonso, J.C., Rodriguez, A., and Gutierrez, C. (1995). Upregulated expression of IL-4 receptors and increased levels of IL-4 in rheumatoid arthritis patients. J. Autoimmun. 8, 587-600.

Schloesser, R.J., Manji, H.K., and Martinowich, K. (2009). Suppression of Adult Neurogenesis Leads to an Increased HPA Axis Response. Neuroreport 20, 553-557.

Schwartz, M., Kipnis, J., Rivest, S., and Prat, A. (2013). How do immune cells support and shape the brain in health, disease, and aging? J. Neurosci. 33, 17587-17596.

Serre, K., Mohr, E., Gaspal, F., Lane, P.J., Bird, R., Cunningham, A.F., and Maclennan, I.C. (2010). IL-4 directs both CD4 and CD8 $\mathrm{T}$ cells to produce Th2 cytokines in vitro, but only CD4 T cells produce these cytokines in response to alum-precipitated protein in vivo. Mol. Immunol. 47, 1914-1922.

Sierra, A., Encinas, J.M., and Maletic-Savatic, M. (2011). Adult human neurogenesis: from microscopy to magnetic resonance imaging. Front Neurosci. 5, 47.
Snyder, J.S., Soumier, A., Brewer, M., Pickel, J., and Cameron, H.A. (2011). Adult hippocampal neurogenesis buffers stress responses and depressive behaviour. Nature 476, 458-461.

Taglialatela, G., Hogan, D., Zhang, W.R., and Dineley, K.T. (2009). Intermediate- and long-term recognition memory deficits in Tg2576 mice are reversed with acute calcineurin inhibition. Behav. Brain Res. 200, 95-99.

Tavakkol Afshari, J., Farid Hosseini, R., Hosseini Farahabadi, S., Heydarian, F., Boskabady, M.H., Khoshnavaz, R., Razavi, A., Ghayoor Karimiani, E., and Ghasemi, G. (2007). Association of the expression of IL-4 and IL-13 genes, IL-4 and IgE serum levels with allergic asthma. Iran J. Allergy Asthma Immunol. 6 , 67-72.

Tsien, J.Z., Huerta, P.T., and Tonegawa, S. (1996). The essential role of hippocampal CA1 NMDA receptor-dependent synaptic plasticity in spatial memory. Cell 87, 1327-1338.

Tsirakis, G., Pappa, C.A., Kaparou, M., Katsomitrou, V., Hatzivasili, A., Alegakis, T., Xekalou, A., Stathopoulos, E.N., and Alexandrakis, M.G. (2011). Assessment of proliferating cell nuclear antigen and its relationship with proinflammatory cytokines and parameters of disease activity in multiple myeloma patients. Eur. J. Histochem. $55,27$.

Walch, L., Massade, L., Dufilho, M., Brunet, A., and Rendu, F. (2006). Pro-atherogenic effect of interleukin-4 in endothelial cells: modulation of oxidative stress, nitric oxide and monocyte chemoattractant protein-1 expression. Atherosclerosis 187, 285291.

Wolf, S.A., Steiner, B., Akpinarli, A., Kammertoens, T., Nassenstein, C., Braun, A., Blankenstein, T., and Kempermann, G. (2009a). CD4-positive T lymphocytes provide a neuroimmunological link in the control of adult hippocampal neurogenesis. J. Immunol. 182, 3979-3984.

Wolf, S.A., Steiner, B., Wengner, A., Lipp, M., Kammertoens, T., and Kempermann, G. (2009b). Adaptive peripheral immune response increases proliferation of neural precursor cells in the adult hippocampus. FASEB J. 23, 3121-3128.

Yirmiya, R., and Goshen, I. (2011). Immune modulation of learning memory, neural plasticity and neurogenesis. Brain Behav. Immunity 25, 181-213.

Zhang, Z., Wang, H., Zhang, G., Hu, D., Xiong, J., Xiong, N., Wang, T., Cao, X., and Mao, L. (2014). Proliferating cell nuclear antigen binds DNA polymerase-beta and mediates 1-methyl-4-phenylpyridinium-induced neuronal death. PLoS One 9, e106669.

Ziv, Y., Ron, N., Butovsky, O., Landa, G., Sudai, E., Greenberg, N., Cohen, H., Kipnis, J., and Schwartz, M. (2006). Immune cells contribute to the maintenance of neurogenesis and spatial learning abilities in adulthood. Nat. Neurosci. 9, 268-275. 